\title{
Detection of EEW wake-up signal with AC parity for ISDB-T television receivers
}

\author{
Satoshi Takahashi ${ }^{\text {a) }}$ \\ Graduate School of Information Sciences, Hiroshima City University, \\ 3-4-1 Ozuka-Higashi, Asa-Minami, Hiroshima 731-3194, Japan \\ a)s.takahashi@m.ieice.org
}

Abstract: When local meteorological agencies advise an emergency alert, ISDB-T (Integrated Services Digital Broadcasting-Terrestrial) television broadcasters would send a special signal to wake up the receivers. There are two ways of waking up: "Emergency Warning System (EWS)" that advises tsunami and earthquake disasters, and "Earthquake Early Warning (EEW)" that advises significant earthquake disasters. For detecting EWS wake-up indications, the use of the parity has been proposed. The method assumed the broadcasters do not change the message except for the wake-up indication. Because we cannot apply the method to an EEW wake-up indication detection, this letter proposes a method for EEW.

Keywords: terrestrial digital television broadcasting, ISDB-T, earthquake early warning, EEW, seismic motion warning

Classification: Terrestrial Wireless Communication/Broadcasting Technologies

\section{References}

[1] Association of Radio Industries and Business (ARIB) ed., Transmission system for digital terrestrial television broadcasting, 2.2 ed., ARIB STD-B31, Tokyo, 2014.

[2] S. J. Choi, "Analysis of emergency alert services and systems," 2007 International Conference on Convergence Information Technology, pp. 657662, Nov. 2007. DOI:10.1109/ICCIT.2007.362

[3] S. Takahashi, "A novel method of determining EWS wake-up trigger for ISDBT digital television receivers," IEEE WiMob 2014 Workshop on Emergency Networks for Public Protection and Disaster Relief (EN4PPDR 2014), Larnaca, pp. 407-412, Oct. 2014. DOI:10.1109/WiMOB.2014.6962193

[4] O. Yamada, "Development of an error-correction method for data packet multiplexed with TV signals," IEEE Trans. Commun., vol. COM-35, no. 1, Jan. 1987. DOI:10.1109/TCOM.1987.1096669

[5] S. Takahashi, "Comparison of two majority determination methods of detecting emergency wake-up trigger for ISDB-T terrestrial digital television receivers," The International Wireless Communications \& Mobile Computing Conference (IWCMC 2015), Dubrovnik, pp. 1-3, Aug. 2015. DOI:10.1109/IWCMC.2015. 7289081 
[6] A. Goldsmith, Wireless Communications, Cambridge University Press, New York, 2005.

\section{Introduction}

If we are advised an emergency alert by a television receiver when disaster occurs, we would realize it promptly and the alert would reduce damages. A mechanism to wake up idle television receivers with a special signal sent from a television broadcaster has been realized. EAS (Emergency Alert System) on ATV (Advanced Television) is in operation in United States. EWS on DVB-T (Digital Video Broadcasting) is used in European countries ${ }^{1}$. EWS and EEW on ISDB-T are available in Japan [1]. And EAS on mobile digital television of T-DMB (Terrestrial Digital Multimedia Broadcasting) in Korea is in service [2].

The ISDB-T digital television standard employs two control signals, and emergency wake-up signals are sent on the control signals. One control signal is TMCC (Transmission and Modulation Configure Control), and it usually tells modulations and error correction redundancies for fixed and mobile receivers. Another is AC (Auxiliary Channel), and it usually transfers the control and timing signals from the broadcasting master station to the broadcasting repeater stations. Both of them are 204-bit length information and employ DBPSK (differential biphase shift keying) modulation. The bit rate is $992 \mathrm{bit} / \mathrm{s}$ and the information is cyclically broadcasting every 0.2 second.

Now, several ISDB-T broadcasters in Japan send the EWS or EEW signals when in emergency situations. An EWS message alerts tsunami and earthquake disasters. When a broadcaster alerts with an EWS message, it set the 26-th bit (excluding the DBPSK phase reference bit) on TMCC so that idle receivers can wake up (that is, the wake-up indication), and the detailed information message is sent on a payload in the transport stream as same as the program such as the videos and audios. The separation of the wake-up indication and the detailed information message enable us to reduce the power consumption of idle receivers. Because for waking up with an EWS message, all an idle receiver has to do is to receive the TMCC signal only. On the other hand, an EEW message alerts earthquakes rapidly rather than an EWS message does. An EEW message consists of both the wake-up indication and the detailed information and is sent on AC.

An ISDB-T digital television broadcaster would send both fixed high-definition videos and mobile low-resolution videos simultaneously. A mobile receiver encounters multipath fading, and it results in a higher bit error rate and also leads to false alarms and misdetections of an EWS wake-up indication. For mobile receivers, a method of reducing false alarms while not increasing misdetection was proposed [3]. The method assumed broadcasters do not change TMCC message except for the EWS wake-up indication. Then, the method used the majority decision of 35 parity bits out of 82 total parity bits. Originally, the parity bits are for the error correction. But the author pointed out a lower false alarm

${ }^{1}$ http: //www.dvb.org/resources/public/factsheets/DVB-EWS-Fact-sheet.pdf 
probability could be achieved with the majority decision rather than the error correction.

An EEW message contains both the wake-up indication and the detailed information of earthquake. Therefore, the AC parity varies due to the detailed information, and the majority decision method is no longer available.

Now, we focus on detecting the wake-up indication of EEW messages. A method of decreasing false alarm of EEW for mobile receivers is proposed in this letter.

\section{Interpreting EEW messages}

\subsection{AC format}

Bit sequence of $\mathrm{AC}$ is cyclically sent, and there is a 16-bit length synchronization word of AC message. In normal situation, $\mathrm{AC}$ is used for a broadcaster. Then television receivers cannot interpret the $\mathrm{AC}$ message, because the synchronization word and the signal format are not disclosed to public.

When a broadcaster advises an EEW message, AC contains synchronizing signal (16-bit-length), start or ending flag (the wake-up indication, 2-bit-length), update flag (2-bit-length), signal identification (3-bit-length), detailed seismic motion warning information (88-bit-length), CRC (10-bit-length), and parity (82bit-length) [1]. The parity is for correcting transmission errors between the start or ending flag and the CRC. Broadcaster identification is set to a part of the detailed information when there is no detailed information available at the broadcaster.

\subsection{Error correction of AC for conventional receivers}

The parity is produced with the $(184,102)$ shorten cyclic error correction codes of the difference-set cyclic codes $(273,191)$ [4]. The code is based on BCH codes, was originally proposed by Weldon, and can be decoded with the majority decision. The generating polynomial is $x^{82}+x^{77}+x^{76}+x^{71}+x^{67}+x^{66}+x^{56}+x^{52}+x^{48}+$ $x^{40}+x^{36}+x^{34}+x^{24}+x^{22}+x^{18}+x^{10}+x^{4}+1$. Because the Hamming weight is 18 (it is equal to the number of the generating polynomial terms), the code can correct error less or equal to 8 bits. The method of correcting errors and the performance analysis under fading can be found in [5] (it is for TMCC, but the same analysis can be applied for the error correction).

\subsection{Bit error rate of DBPSK modulated signal in fading environments}

A bit error rate, $P_{e}$, of DBPSK in AWGN (additive white Gaussian noise) is known and it is [6]

$$
P_{e}=\frac{1}{2} \exp (-\gamma)
$$

where $\gamma$ is energy per 1 bit relative to the noise power density and it is often referred to as $E_{b} / N_{0}$. A mobile receiver would encounter Rayleigh fading and $P_{e}$ is

$$
P_{e}=\frac{1}{2}\left[\frac{1+\gamma\left(1-\rho_{C}\right)}{1+\gamma}\right],
$$




$$
P_{e}=\frac{1}{2}\left(1-\rho_{C}\right)
$$

\section{Proposed detection of wake-up indication with majority decision}

As described in Sect. 2.1, there is various information such as the detailed seismic motion warning information in $\mathrm{AC}$ when an EEW message is advised.

However, most of all information bits is disused for the end situation of an EEW message, because the broadcaster no longer does not have to send the specific information. Therefore, all EEW messages except for the end situation are assumed to be EEW wake-up indication here. The proposed method can be described with following steps:

1. the receiver is waiting for an EEW synchronization word on $\mathrm{AC}$,

2. the receiver counts the number of active bits among the start or ending flag and the specific parity bits,

3. if the number is less than a threshold, it is not the end situation and the receiver wakes up.

To do this, the parity bit sequence with the broadcaster identification should be calculated so that receivers can know the end situation.

When the end situation is advised, the start or ending bits are 00 , the signal identification bits are also 000 . Then, the parity bits of $x^{80}, x^{77}, x^{72}, x^{67}, x^{65}, x^{60}, x^{57}$, $x^{55}, x^{52}, x^{51}, x^{48}, x^{46}, x^{41}, x^{40}, x^{35}, x^{34}, x^{32}, x^{30}, x^{26}, x^{25}, x^{22}, x^{20}, x^{19}, x^{16}, x^{15}, x^{12}$, $x^{11}, x^{10}, x^{8}, x^{5}, x^{3}$ indicate the specific value defined by the broadcaster identification. Therefore, there are 36 bits available for the majority decision.

The performance is evaluated in terms of misdetection probability $\left(P_{\mathrm{md}}\right)$ and false alarm probability $\left(P_{\mathrm{fa}}\right) . P_{\mathrm{md}}$ is the complementary probability the 18 synchronization bits are received correctly and the number of the agree bits of the 36 bits is less than 18 ,

$$
P_{\mathrm{md}}^{\mathrm{prop}}=1-\left(1-P_{e}\right)^{16} \cdot\left\{\sum_{k=0}^{18}{ }_{36} C_{k}\left(1-P_{e}\right)^{36-k} \cdot P_{e}^{k}\right\} .
$$

On the other hand, $P_{\mathrm{fa}}$ is the probability the synchronization bits are received correctly and the more than the 19 bits out of the 36 bits disagree,

$$
P_{\mathrm{fa}}^{\mathrm{prop}}=\left(1-P_{e}\right)^{16} \cdot\left\{\sum_{k=19}^{36}{ }_{36} C_{k}\left(1-P_{e}\right)^{36-k} P_{e}^{k}\right\} .
$$

These probabilities for the conventional method of using error correction for the parity can be obtained as the same way. $P_{\mathrm{md}}$ is the complementary probability the synchronization bits and the start or ending bits are correctly received (each of the latter probability is the number of error is less than 8 bits for the 17 bits with the error probability of $P_{e}$, see [5]),

$$
P_{\mathrm{md}}^{\mathrm{conv}}=1-\left(1-P_{e}\right)^{16} \cdot\left\{\sum_{k=0}^{8}{ }_{17} C_{k}\left(1-P_{e}\right)^{17-k} \cdot P_{e}^{k}\right\}^{2} .
$$

() IEICE 2016 DOI: 10.1587/comex.2016XBL0068 Received March 29, 2016 Accepted April 12, 2016 Publicized April 22, 2016 Copyedited July 1, 2016 


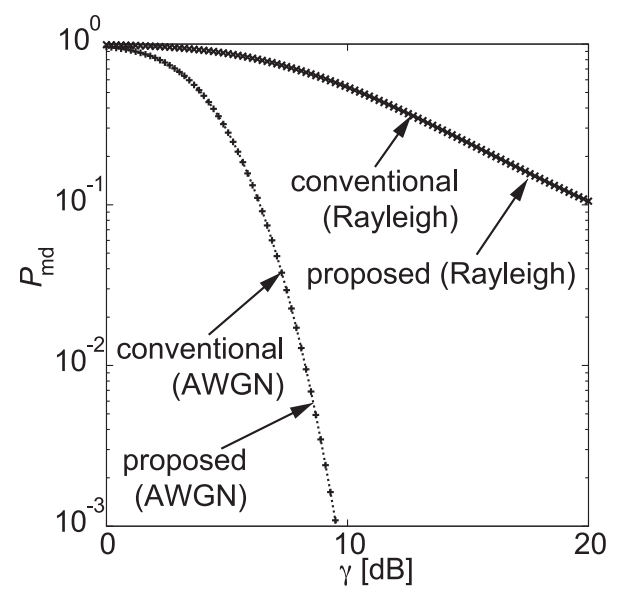

(a)

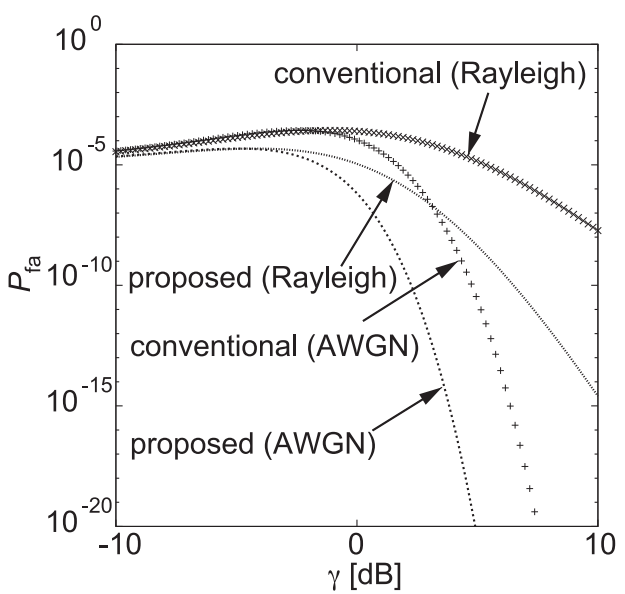

(b)

Fig. 1. Misdetection probability (a) and false alarm probability (b) in AWGN and Rayleigh fading environments.

$$
P_{\mathrm{fa}}^{\mathrm{conv}}=\left(1-P_{e}\right)^{16} \cdot\left[1-\left\{\sum_{k=0}^{8}{ }_{17} C_{k}\left(1-P_{e}\right)^{17-k} P_{e}^{k}\right\}^{2}\right] .
$$

\section{Performance evaluation}

A center frequency of $600 \mathrm{MHz}$ was assumed in the evaluation. Performance of the conventional and proposed methods is compared in both AWGN and Rayleigh fading environments.

$P_{\mathrm{md}}$ of both the conventional and proposed method in AWGN are calculated substituting Eq. (1) with Eqs. (4) and (6). $P_{\mathrm{md}}$ in Rayleigh fading environments are also obtained using Eqs. (2), (6), and (4) by assuming the velocity of $10 \mathrm{~m} / \mathrm{s}$. They are plotted in Fig. 1(a) as a function of $E_{b} / N_{0}(\gamma) . P_{\mathrm{md}}$ decreased as an increase in $\gamma$. This figure also showed that $P_{\mathrm{md}}$ for the proposed method was almost same as $P_{\mathrm{md}}$ for the conventional method. $P_{\mathrm{md}}$ in Rayleigh fading environment was always higher than $P_{\mathrm{md}}$ in AWGN for an entire range of $\gamma$, because $P_{e}$ in Rayleigh fading environments always was higher than $P_{e}$ in AWGN. The proposed method did not increase $P_{\mathrm{md}} . P_{\mathrm{fa}}$ for the conventional and proposed methods are also compared using Eqs. (1), (2), (5), and (7). $P_{\mathrm{fa}}$ as a function of $\gamma$ is plotted in Fig. 1(b). $P_{\mathrm{fa}}$ showed convex shape as an increase in $\gamma \cdot P_{\mathrm{fa}}$ in Rayleigh fading environment was higher than $P_{\mathrm{fa}}$ in AWGN as same as the case of $P_{\mathrm{md}}$. According to the Fig. 1(b), $P_{\mathrm{fa}}$ for the proposed method is lower than $P_{\mathrm{fa}}$ for the conventional method at almost all regions of $\gamma$. For example in the Rayleigh fading environments at $\gamma$ of $10 \mathrm{~dB}, P_{\mathrm{fa}}$ for the conventional and proposed methods were $1.37 \times 10^{-8}$ and $1.35 \times 10^{-15}$. Because AC is sent about 5 frames in a second, the false alarm corresponds the conventional method wrongly alerts an EEW wake-up indication about every 168 days, but the proposed method do more than $4.68 \times 10^{6}$ years.

For comparing the conventional and proposed methods, $P_{\mathrm{fa}}$ as a function of $P_{\mathrm{md}}$ is calculated and shown in Fig. 2. $P_{\mathrm{fa}}$ for the proposed method was lower than that for the conventional method at the same $P_{\mathrm{md}}$. Especially for a lower $P_{\mathrm{md}}$ region (a higher $\gamma$ ), the proposed method significantly reduced $P_{\mathrm{fa}}$. 


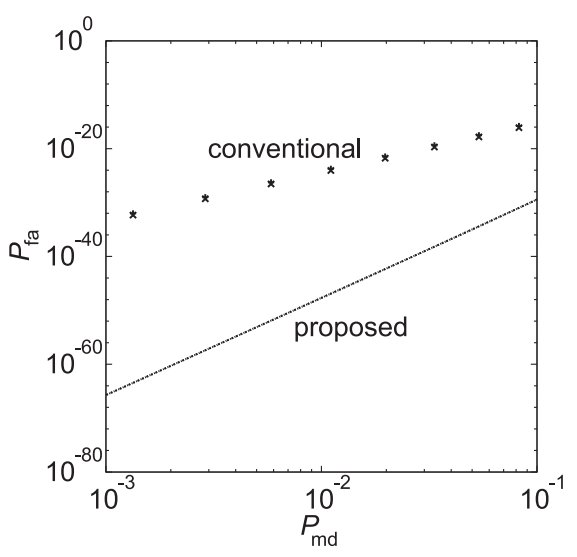

Fig. 2. False alarm probability as a function of misdetection probability.

\section{Conclusion}

In this paper, the method of receiving ISDB-T EEW signals for mobile television receivers was proposed. The proposed method focused on detecting the wake-up indication. Because there are few information sent when the end situation of an EEW message is advised, a receiver of the proposed method tries to detect the end situation of the EEW message and alerts when the message is not end one. The detection could be accomplished by majority decision of specific bits including parity bits. The result showed the proposed method significantly reduced the false alarm probability while did not increase the misdetection probability.

\section{Acknowledgment}

This work was supported by JSPS KAKENHI Grant Number 26420368 and the Hiroshima City University Grant for Special Academic Research (General Studies). 